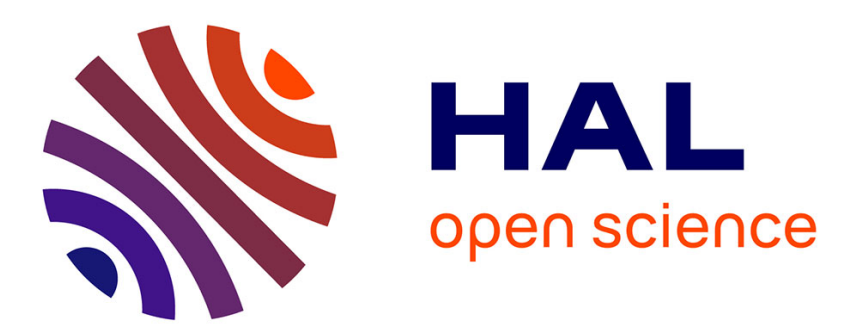

\title{
Photopyroelectric study of the thermal parameters of antiferromagnets at the Neel temperature
}

M. Marinelli, F. Mercuri, U. Zammit, R. Pizzoferrato, F. Scudieri, D. Dadarlat

\section{To cite this version:}

M. Marinelli, F. Mercuri, U. Zammit, R. Pizzoferrato, F. Scudieri, et al.. Photopyroelectric study of the thermal parameters of antiferromagnets at the Neel temperature. Journal de Physique IV Proceedings, 1994, 04 (C7), pp.C7-261-C7-266. 10.1051/jp4:1994762 . jpa-00253291

\section{HAL Id: jpa-00253291 https://hal.science/jpa-00253291}

Submitted on 1 Jan 1994

HAL is a multi-disciplinary open access archive for the deposit and dissemination of scientific research documents, whether they are published or not. The documents may come from teaching and research institutions in France or abroad, or from public or private research centers.
L'archive ouverte pluridisciplinaire HAL, est destinée au dépôt et à la diffusion de documents scientifiques de niveau recherche, publiés ou non, émanant des établissements d'enseignement et de recherche français ou étrangers, des laboratoires publics ou privés. 


\title{
Photopyroelectric study of the thermal parameters of antiferromagnets at the Neel temperature
}

\author{
M. Marinelli, F. Mercuri, U. Zammit, R. Pizzoferrato, F. Scudieri and D. Dadarlat* \\ Dipartimento di Ingegneria Meccanica - II, Università di Roma "Tor Vergata", Via della Ricerca Scientifica, \\ 00173 Roma, Italy \\ * Institute of Isotopic and Molecular Technology, P.O. Box 700, Cluj-Napoca 5, 3400, Romania
}

\begin{abstract}
High resolution measurements have been performed with the photopyroelectric technique to study the static and dynamic critical behaviour of the thermal parameters of the two antiferromagnets $\mathrm{Cr}_{2} \mathrm{O}_{3}$ and $\mathrm{FeF}_{2}$ close to the Neel temperature. Specific heat, thermal diffusivity and thermal conductivity behaviours have been simultaneously measured in the critical region of the second order antiferromagnetic-paramagnetic phase transition. Critical exponents and the critical amplitude ratios of specific heat and thermal diffusivity have been determined. We have also studied single crystal Chromium for which the antiferromagnetic - paramagnetic phase transition is first order. The effect of thermal annealing on the signal amplitude and phase in the vicinity of the transition has been reported.
\end{abstract}

\section{INTRODUCTION}

Two theoretical models have been introduced to describe the static critical phenomena associated with the antiferromagnetic - paramagnetic phase transition of the antiferromagnets: the Heisenberg model which applies to isotropic systems and the Ising model which is valid for uniaxial systems [1]. Each model predicts particular values for the critical exponents of the specific heat and for the amplitudes ratio of the critical term.

A manifold of experimental data regarding the specific heat are available in the literature. For some materials such as $\mathrm{FeF}_{2}$ the results are consistent with the Ising model [2], while in the case of $\mathrm{Cr}_{2} \mathrm{O}_{3}$, for example, there are no definitive results reported [3]. This could be due to the possibility of a crossover from Heisenberg to Ising models as the transition temperature is approached. The situation is completely different for the dynamic thermal quantities for which not enough high resolution measurements are available in literature. In the case of $\mathrm{Cr}$, since the transition is a first order one, it is not possible to calculate the thermal parameters from the data. It is possible however to monitor the effect of the annealing process on the sample via the monitoring of the signal amplitude and phase. This is very important because of the strong dependence of the behaviour of $\mathrm{Cr}$ samples [4] close to the transition upon the residual stress present in the sample.

The application of the photopyroelectric technique (PPE) for the study of the magnetic critical phenomena can greatly improve the knowledge in this field. It allows the simultaneous measurement of the specific heat, the thermal diffusivity and the thermal conductivity. This simultaneous study is essential for a deep understanding of the nature of a phase transition. Moreover the high resolution of the technique allows investigation of rather small reduced temperature regions around $T_{N}$. It is, therefore, possible to 
reveal variations in the critical exponent value when different temperature ranges are considered so indicating the presence of a crossover.

\section{THEORY}

The photopyroelectric technique, in the standard back detection configuration, consists (see Fig. 1) of a sample with one side put in thermal contact with a pyroelectric transducer while the opposite side is periodically heated by an incident modulated laser beam.

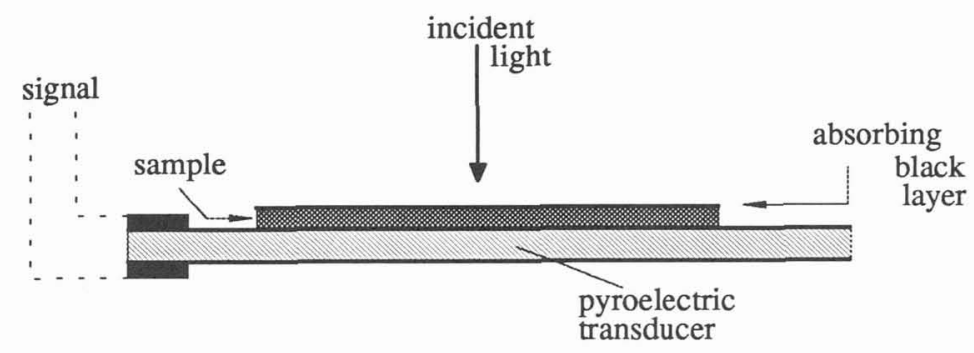

Fig. 1 Photopyroelectric cell

If the sample is opaque and thermally thick (thermal diffusion length much less than the thickness) and the pyroelectric is thermally thick, both being optically opaque, and if $\beta \sqrt{ }\left(\omega / 2 D_{s}\right)>>1$ ( $\beta$ being the optical absorption coefficient) the theory predicts for the generated pyroelectric signal, the following expressions of amplitude and phase:

$$
V \propto \frac{1}{\sqrt{1+\omega^{2} \tau_{e}^{2}}} \frac{e^{-\sqrt{\frac{\omega}{2 D_{s}}} L_{s}}}{\left(e_{s}+e_{m}\right)\left(e_{p} / e_{s}+1\right)} ; \quad \varphi=\operatorname{tg}^{-1}\left(-\omega \tau_{e}\right)-\sqrt{\frac{\omega}{2 D_{s}}} L_{s} ;
$$

where $\omega=2 \pi f$; $f$ is the modulation frequency; $\tau_{e}$ is inverse of the electrical cut-off frequency; $e_{p}$ and $e_{m}$ are the effusivity of pyroelectric and front medium respectively; $D_{s}, e_{s}$ and $L_{s}$ are the thermal diffusivity, the effusivity and the thickness of the sample. For a given $L_{s}$ and $\omega$, knowing the $e_{p}$ and $e_{m}$, it is possible to calculate $\mathrm{D}_{\mathrm{s}}$ and $\mathrm{e}_{\mathrm{s}}$. Knowing the sample density $\rho_{\mathrm{s}}$, one can obtain the thermal conductivity $k_{s}=e_{s} \sqrt{D_{s}}$ and the specific heat $c_{s}=e_{s} / \rho_{s} \sqrt{D_{s}}$. Moreover, at a fixed temperature, when $\omega$ is high enough to make $\omega \tau_{\mathrm{e}} \ll<1$ and $\operatorname{tg}^{-1}\left(-\omega \tau_{\mathrm{e}}\right)$ approximately constant, we have:

$$
V \propto\left(-\sqrt{\frac{\pi}{D_{s}}} L_{s}\right) \sqrt{f} ; \quad \varphi \propto\left(-\sqrt{\frac{\pi}{D_{s}}} L_{s}\right) \sqrt{f} ;
$$

There is hence in such conditions a linear dependency of amplitude and phase on the square root of frequency.

\section{EXPERIMENTAL}

In the present paper measurements around the Neel temperature on two different antiferromagnets are reported. The first one, $\mathrm{FeF}_{2}$, has its transition at low temperature $(78.4 \mathrm{~K})$, while the latter, $\mathrm{Cr}_{2} \mathrm{O}_{3}$, is at room temperature ( about $308 \mathrm{~K}$ ). It was therefore necessary to use two different experimental set-ups.

To measure $\mathrm{FeF}_{2}$, a low temperature set-up was used. The back side of the pyroelectric, as shown in Fig.1, was glued on a copper holder whose bottom was in thermal contact with the cold finger of a cryocooler. The entire sample holder was in vacuum. The opacity of the sample was ensured by a black 
thermally thin absorbing layer deposited on its front surface. In this case the sample was $620 \mu \mathrm{m}$ thick and the modulation frequency of the heating laser beam was $27 \mathrm{~Hz}$. The incident beam light source was an HeNe laser emitting at $\lambda=633 \mathrm{~nm}$. The heating rate was $10 \mathrm{mK} / \mathrm{min}$ and the data were collected every $10 \mathrm{mK}$.

In the case of $\mathrm{Cr}_{2} \mathrm{O}_{3}$, the $350 \mu \mathrm{m}$ thick sample was put in thermal contact with the pyroelectric transducer as shown in Fig.1, by means of a silicon grease layer as coupling fluid. The layer was thermally thin so that we could neglect its influence on the thermal transport process. The pyroelectric transducer was in both cases a $300 \mu \mathrm{m}$ thick lithium tantalate single crystal. Everything was placed in a copper sample holder contained in an oven. By means of a thermistor placed in the sample holder very close to the sample, we could control the temperature and the heating rate. The working heating rate was about $(4 \pm 1) \mathrm{mK} / \mathrm{min}$ and the data were collected every $2 \mathrm{mK}$. The laser beam was modulated at $78 \mathrm{~Hz}$ for which frequency the sample and pyroelectric were thermally thick.

In the case of single crystalline Chromium a sample $960 \mu \mathrm{m}$ thick sample was used. The experimental set up was the same as for $\mathrm{Cr}_{2} \mathrm{O}_{3}$ since the transition temperature was about $311 \mathrm{~K}$. This transition is known to be first order while the first two are known to be second order.

Frequency scans have been performed for each sample to check experimentally that at the given modulation frequencies the required conditions were completely fulfilled. These measurements are shown in Fig. 2 where the products $\mathrm{f} \times \mathrm{V}$ on logarithmic scale and the phase in radians are plotted versus $\sqrt{\mathrm{f}}$. According the expressions (2), a linear behaviour is clearly evident. We choose the modulation frequency for the measurements well within the linear region. An absolute determination of the thermal diffusivity is possible from the slope of the two curves. Within the experimental errors, we obtained the same value, reported in the figure, from the amplitude and phase
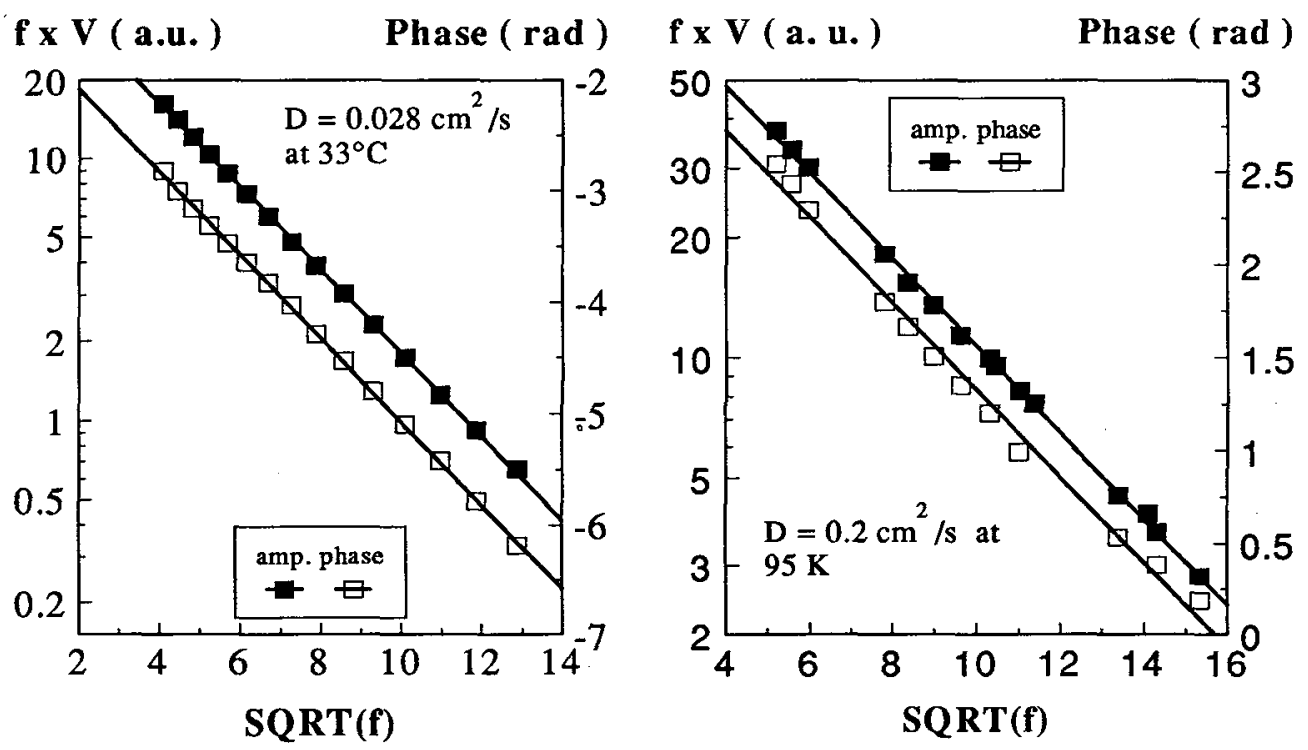

Fig. 2 Amplitude times frequency on logarithmic scale and phase vs. square root of frequency for (a) $\mathrm{Cr}_{2} \mathrm{O}_{3}$ and (b) $\mathrm{FeF}_{2}$ respectively. The thermal diffusivity absolute values obtained from the slopes are reported on the graph. 


\section{RESULTS}

Fig. 3 shows the specific heat, thermal diffusivity and thermal conductivity of $\mathrm{FeF}_{2}$ near the transition temperature. A divergence in the specific heat and a dip in thermal diffusivity are clearly evident. No anomalies, but a small discontinuity has been found in the thermal conductivity.

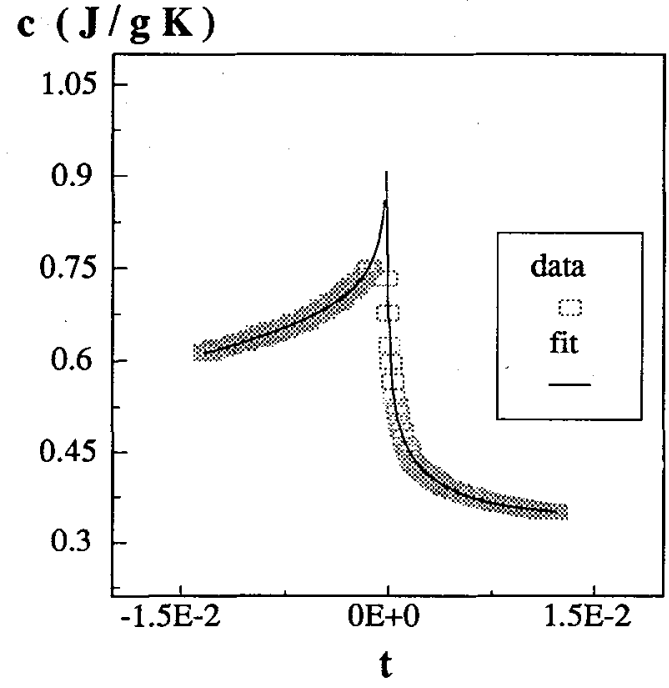

(a)

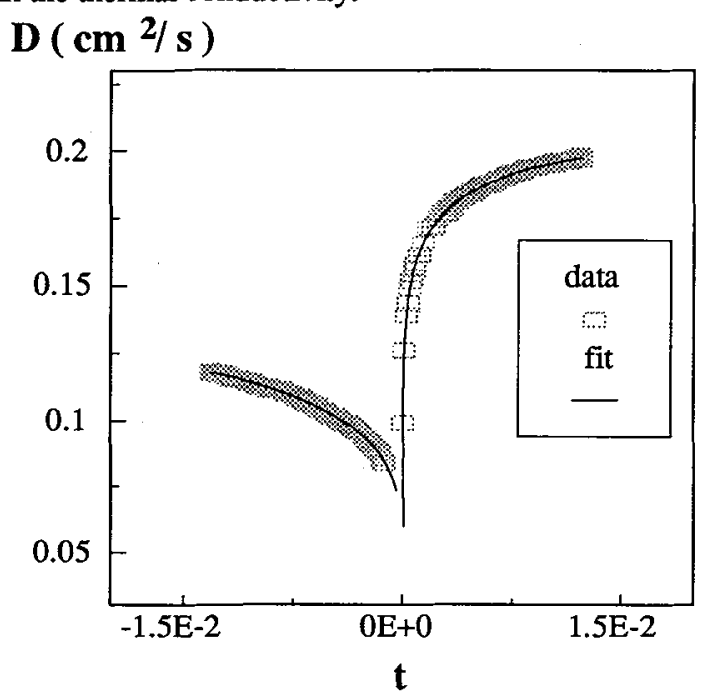

(b)

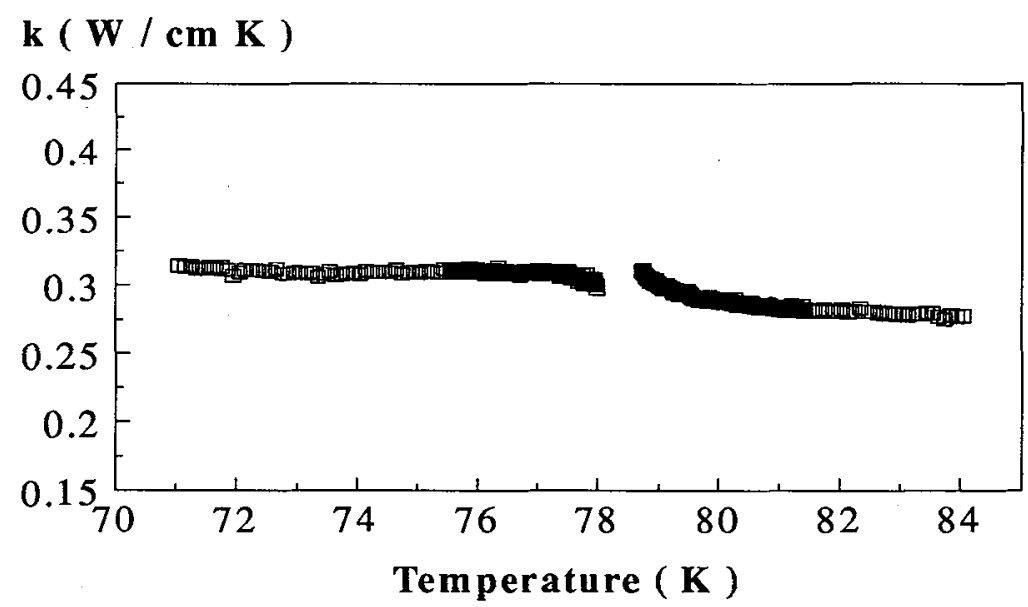

(c)

Fig. 3 Data and fits of specific heat (a), thermal diffusivity (b) vs. reduced temperature $t=\mid \mathrm{T}-\mathrm{Tcl} / \mathrm{Tc}$ and thermal conductivity data (c) vs. temperature of $\mathrm{FeF}_{2}$

It is well known [4] that the static critical behaviour of $\mathrm{FeF}_{2}$ can be described by the Ising model which predicts for the critical exponent of the specific heat a value of $\alpha=0.115$. We fitted the data with the usual expression 
$c=B+E t+A|t|^{-\alpha}\left(1+D|t|^{0.5}\right)$

where $A, B, E, D$ and $\alpha$ were the fitting parameters, $t=T-T_{c}$ being the reduced temperature. Data in the reduced temperature range $0.013-0.001$ were included in the fit and we obtained $\alpha=0.102$ which is in good agreement with the theoretical predictions. No experimental data can be found in the literature for the thermal transport properties. We tried to interpret the thermal diffusivity with dynamic models which are the analogue of the Ising model for dynamics. There are two possibilities which depends on the energy conservation of the spin system: if the energy is conserved (mod C) the theory predicts for the critical exponent of the diffusivity $-b=\alpha=0.115$, while if the energy is not conserved (mod. A) $b-0.015$. We fitted the data in the same reduced temperature interval of the specific heat with the function

$D=V+W t+U|t|^{-b}\left(1+F|t|^{05}\right)$

and the value we obtained was $b=-0.128$ which is in good agreement with model $C$.

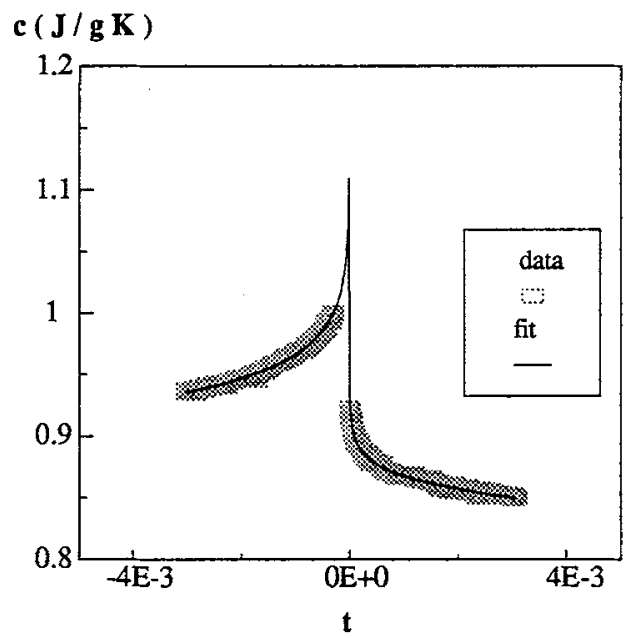

(a)

$$
D\left(\mathrm{~cm}^{2} / \mathrm{s}\right)
$$

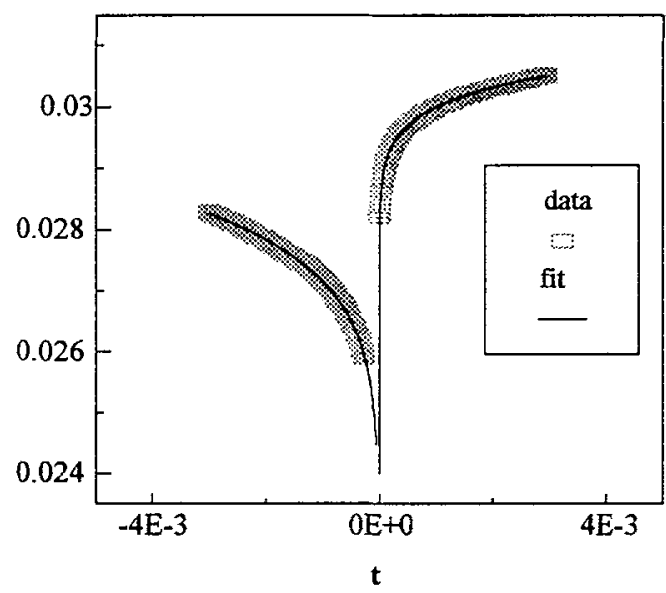

(b)

\section{$\mathbf{k}(\mathbf{W} / \mathbf{c m ~ K})$}

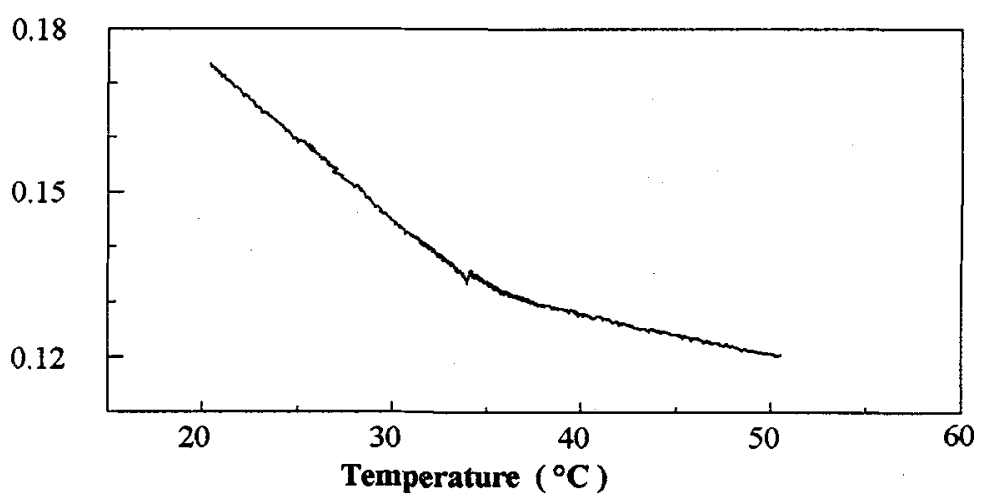

(c)

Fig. 4 Data and fits of specific heat (a), thermal diffusivity (b) vs. reduced temperature $\mathrm{t}=\mid \mathrm{T}-\mathrm{Tcl} / \mathrm{Tc}$ and thermal conductivity data (c) vs. temperature of $\mathrm{Cr}_{2} \mathrm{O}_{3}$

Fig. 4 shows the specific heat, thermal diffusivity and thermal conductivity of $\mathrm{Cr}_{2} \mathrm{O}_{3}$. No conclusive data can be found in literature for the critical behaviour of this material even for the specific heat. It is essentially due to the possibility of cross over from the Heisenberg behaviour (isotropic) to an Ising-like 
one very close to $T_{c}$. For this reason a high resolution technique which allows measurements very close to the transition temperature is extremely important. We fit first the specific heat data in a wide reduced temperature range around $T_{c}(0.008<t<0.0001)$ and we obtained $\alpha=-0.032$ which does not agree neither with Heisenberg nor with Ising. If only data points in $t$ range $0.003-0.00005$ were included in the fit, we obtain a value of $\alpha=0.1$ which is in a good agreement with the Ising value of 0.115 . This confirm that a crossover behaviour is present in this material. We also fit the thermal diffusivity data and in the $t$ range where the Ising predictions were valid we found an exponent $b=-0.09$ which again is in agreement with the model C predictions.

Fig, 5 shows the amplitude and phase close to the transition temperature of the antiferromagnetic paramagnetic phase transition of single crystalline $\mathrm{Cr}$. The critical behaviour of this material is strongly affected by the presence of thermal stresses in the sample.

Amplitude (a. u. )

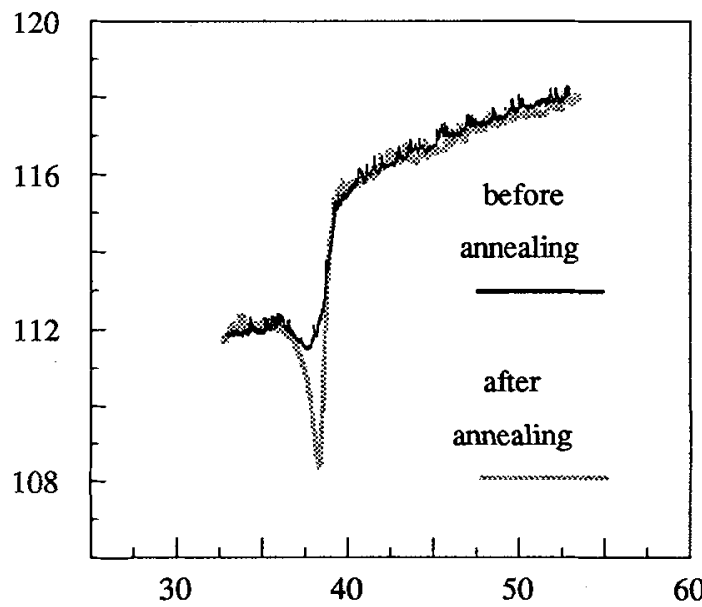

Temperature $\left({ }^{\circ} \mathrm{C}\right.$ )

\section{Phase $\left(^{\circ}\right.$ )}

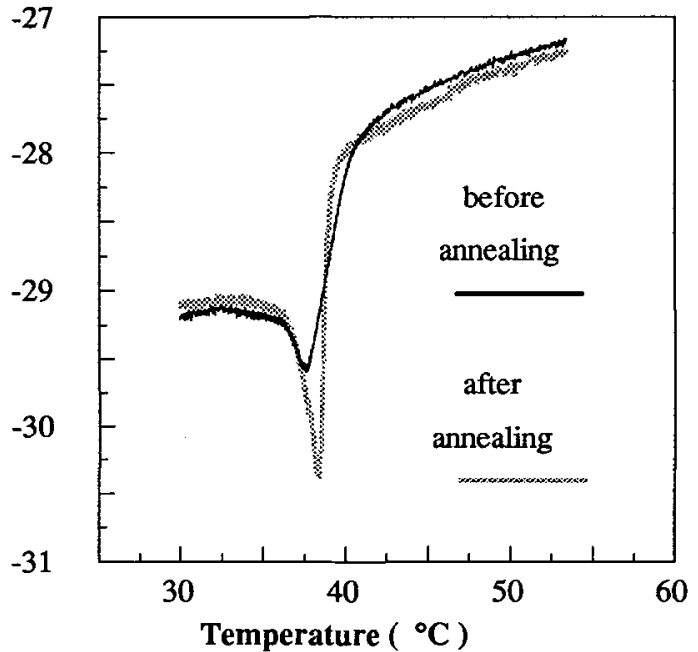

Fig. 5 Chromium amplitude and phase vs. temperature at the magnetic transition before and after the annealing respectively

It is clearly evident in the figures the effect of the annealing which has been made to partially remove defects. The sample was annealed at $850{ }^{\circ} \mathrm{C}$ for 60 hours and then allowed to cool down to room temperature in 24 hours. The annealing was made in Argon.

The above results clearly demonstrate that the photopyroelectric technique is suitable for high resolution studies close to the phase transition. It allows not only the study of static critical phenomena as in the case of the specific heat, but also a high resolution study of the critical dynamics of the systems under investigation.

\section{References}

[1]. Hohenberg P.C., Halperin B.I., Rev. Mod. Phys., 49, 435 (1977)

[2]. Bruce R.H., Cannell D.S., Phys. Rev. B, 15, 4451 (1977)

[3]. Chirwa M., Lundgren L., Nordblad P. and Beckman O., J. Magn. Magn. Mater., 15-18 457 (1980)

[4]. Williams I.S., Gopal E.S.R. and Street R., J. Phys. F: Metal Phys, 9 (3), 431 (1979) 